\title{
Minimization of scalar curvature in conformal geometry
}

\author{
Zisis N. Sakellaris ${ }^{1}$ (D)
}

Received: 13 April 2016/ Accepted: 22 July 2016 / Published online: 11 August 2016

(C) The Author(s) 2016. This article is published with open access at Springerlink.com

\begin{abstract}
Let $\left(M, g_{0}\right)$ a smooth compact Riemannian manifold with smooth boundary and dimension $n \geq 3$. We consider a minimization problem for the scalar curvature $R$ after a conformal change. In particular, we seek for minimizers of the $\|\cdot\|_{\infty}$ functional of $R$, within a conformal class, under small energy assumptions and natural geometric constraints. We prove that minimizers exist, and have locally constant scalar curvature, outside of a set $\Gamma$ with explicit description.
\end{abstract}

Keywords Scalar curvature $\cdot$ Variational problem · Conformal geometry

\section{Introduction: statement of the main result}

Let $\left(M, g_{0}\right)$ a smooth compact Riemannian manifold of dimension $n \geq 3$, having smooth boundary, equipped with a smooth Riemannian metric $g_{0}$ and corresponding scalar curvature $R_{0}$. If we let $u: \bar{M} \rightarrow \mathbb{R}$ be a smooth positive function, we may consider conformal changes of the metric, having the form:

$$
g=u^{2^{*}-2} g_{0},
$$

where $2^{*}=\frac{2 n}{n-2}$ is the critical exponent for the Sobolev embedding $W^{1,2} \hookrightarrow L^{p}$. Recall that the scalar curvature of $g_{0}$ transforms under the law:

$$
-c_{n} \Delta_{g_{0}} u+R_{0} u=R_{g} u^{2^{*}-1}
$$

(see [12] for example), where $\Delta_{g_{0}}$ is the Laplace-Beltrami operator of $g_{0}, c_{n}=\frac{4(n-1)}{(n-2)}$ and $R_{g}$ is the scalar curvature of $g$.

Equation (2) has been the object of intense study in the past, beginning with Yamabe [20], who claimed that solutions $u$ exist, for $R_{g}$ constant, on any closed manifold. Nevertheless, Trudinger [19] found a gap in the proof and proved part of the original statement, with Aubin

Zisis N. Sakellaris

znsakell@hotmail.com

1 Department of Mathematical Sciences, University of Bath, Bath BA2 7AY, UK 
[1] and Schoen [16] completing the proof in the remaining cases. A unified approach for the Yamabe problem can be found in [12]. Furthermore, similar results were obtained in the case of manifolds with boundary, beginning with the work of Escobar [4,5], and continuing with Marques [13,14].

Another related problem to that of Yamabe is that of prescribed scalar curvature, known as the Nirenberg problem for surfaces. In that context, it is asked if a certain smooth function can be the scalar curvature function of a Riemannian manifold, after a conformal change (see $[3,11]$ for example). We note that obstructions may exist, depending on the manifolds and functions that are studied.

A different question, connected to the aforementioned results, was raised in [15]. In particular, a minimization problem for a weighted variant of the Gaussian curvature, after a conformal change, was studied, on a compact smooth surface $\left(S, g_{1}\right)$ with smooth boundary $\partial S$. If $g_{2}$ is a metric conformal to $g_{1}$, with $g_{2}=e^{2 f} g_{1}$ for a smooth function $f$, we have the Gaussian curvature of $g_{2}, K$, given by:

$$
K=e^{-2 f}\left(-\Delta_{g_{1}} f+K_{g_{1}}\right),
$$

where $\Delta_{g_{1}}$ is the Laplace-Beltrami operator of $g_{1}$ and $K_{g_{1}}$ is the scalar curvature of $g_{1}$. After the introduction of a smooth positive weight function $k: S \rightarrow \mathbb{R}$, the authors prove that the functional:

$$
E_{\infty}(f)=\operatorname{ess} \sup _{S} \frac{|K|}{k}
$$

attains its infimum on a suitably defined set. This is valid, provided that certain bounds hold on the energy of the minimizer, in the class of functions they study. Moreover, the Gaussian curvature of the minimizer is locally constant, outside of a closed set $\Gamma$, providing a certain connection to the prescribed Gaussian curvature problem.

Motivated by the aforementioned results from [15] in the surface case, we study a higherdimensional analogue of that problem. In particular, the question that we attempt to answer is the following:

Is there a metric in the conformal class of $g_{0}$, with scalar curvature $R$ minimizing the $L^{\infty}$ norm?

Hence, we ask whether the following infimum is attained:

$$
\inf _{g \in\left[g_{0}\right]}\left\|R_{g}\right\|_{L^{\infty}(M)}
$$

where $\left[g_{0}\right]$ is the class of metrics pointwise conformally equivalent to $g_{0}$. Since we are considering a variational problem in a geometric setting, there is always the possibility of the problem admitting a trivial solution. First, note that for any $\lambda \in \mathbb{R}$, we can consider the metric $g_{\lambda}=(\lambda u)^{2^{*}-2} g_{0}$, with curvature $R^{\lambda}$ given by:

$$
R^{\lambda}=\lambda^{2-2^{*}} R_{g}
$$

Hence, because of this scaling property, the infimum we want to consider would be automatically zero, or not attained. To avoid this, we fix the volume of our manifold with respect to $g$. If $\mu_{0}$ is the measure corresponding to the metric $g_{0}$, and $\mu$ that is corresponding to $g$, then the relation $\mu=u^{2^{*}} \mu_{0}$ holds. Thus, we select a number $c_{1} \in \mathbb{R}$, with $0<c_{1}$, and require that:

$$
\mu(M)=c_{1} .
$$


If $h_{0}$ and $h_{g}$ are the mean curvature functions of $\partial M$ for $g$ and $g_{0}$ respectively, after a conformal change of the form (1), then the following equation holds:

$$
\frac{n-2}{2} h_{g} u^{\frac{n}{n-2}}=\frac{n-2}{2} h_{0} u+\frac{\partial u}{\partial v_{0}},
$$

with $v_{0}$ standing for the outward unit normal to $\partial M$ with respect to $g_{0}$. Taking advantage of the last formula, we select a number $c_{2} \in \mathbb{R}$ such that:

$$
\int_{\partial M} h_{g} \mathrm{~d} \sigma=\int_{\partial M} h_{g} u^{\frac{2(n-1)}{n-2}} \mathrm{~d} \sigma_{0}=c_{2} .
$$

Here $\sigma$ is the surface measure corresponding to $g$, and $\sigma_{0}$ that corresponding to $g_{0}$ as usual. In that way we may avoid the possibility of the presence of a scalar-flat metric in a conformal class, which follows from standard results of Escobar [4], extended by Marques in [13] and [14]. Finally, we also prescribe $u$ along the boundary, with:

$$
u=u_{0} \in C^{\infty}(\partial M),
$$

for a fixed positive function $u_{0}$.

After having given the necessary constraints for our purposes, we can now proceed to specifying some subsets of Sobolev spaces to work with. For $\frac{n}{2}<p<\infty$, we define the subset $A^{p}\left(c_{1}, c_{2}, u_{0}\right)$ of the Sobolev space $W^{2, p}\left(M, g_{0}\right)$ by:

$$
\begin{aligned}
A^{p}\left(c_{1}, c_{2}, u_{0}\right)= & \left\{u \in W^{2, p}\left(M, g_{0}\right), \int_{M} u^{2^{*}} \mathrm{~d} \mu_{0}=c_{1},\right. \\
& \left.\int_{\partial M} h_{g} \mathrm{~d} \sigma=c_{2}, u=u_{0} \text { on } \partial M, u>0\right\} .
\end{aligned}
$$

We also let $A^{\infty}\left(c_{1}, c_{2}, u_{0}\right)$ be the set of all $u \in \bigcap_{p<\infty} A^{p}\left(c_{1}, c_{2}, u_{0}\right)$, with scalar curvature $R \in L^{\infty}\left(M, \mu_{0}\right)$. We will show that within $A^{\infty}$, there exists a minimizer for our problem, as long as an upper bound on the infimum of the energy $E(u)$ is satisfied.

In particular, with this notation in hand, our main result is as follows:

Theorem 1 Let $\left(M, g_{0}\right)$ a smooth compact Riemannian manifold with smooth boundary and dimension $n \geq 3$. Let $u_{0} \in C^{\infty}(\partial M), c_{1}>0, c_{2} \in \mathbb{R}$, satisfy

$$
\inf _{u \in A^{\infty}\left(c_{1}, c_{2}, u_{0}\right)} E(u)<\frac{c_{n}}{c_{1}^{2 / n} K_{n}^{2}},
$$

with $K_{n}$ being the best constant for the Euclidean Sobolev inequality and $c_{n}=\frac{4(n-1)}{n-2}$. Then, a minimizer $u$ of $E$ in $A^{\infty}\left(c_{1}, c_{2}, u_{0}\right)$ exists, with scalar curvature $R$ satisfying $|R|=E(u)$, almost everywhere. Moreover, $R$ is locally constant in $M \backslash \Gamma$, where $\Gamma$ is a closed set contained in a countable union of embedded $(n-1)$-dimensional $C^{1, \rho}$ submanifolds and a closed $(n-2)$-dimensional set.

This result is in line with the corresponding result in the case of surfaces in [15]. On the other hand, since the transformation equation (2) involves a different kind of nonlinearity compared to (5), different methods are needed to prove existence of a minimizer. In addition, the Gauss-Bonnet Theorem, which holds in a generalized sense for $n \geq 3$, does not restrict our constraints directly, something characteristic of the surface case. Finally, we remark that it is natural to impose some bounds on the infimum of $E(u)$, with similar conditions having been used in related problems, see [12] or [17] for example. 
An interesting phenomenon is that the set $\Gamma$, which is countably $(n-1)$ rectifiable, as can be seen using standard results from [18] for example, has another representation. It is the nodal set of the solution of a partial differential equation related to our minimization problem. Remarking that we have:

$$
E(u)=|R|,
$$

almost everywhere for our minimizer $u$, we can see that we recover a metric with constant scalar curvature, locally up to sign, outside of a set with $\mu_{0}(\Gamma)=0$. Moreover, (6) still holds for the minimizer over the boundary. Nevertheless, our constraints may prevent $\Gamma$ from being empty, as we have already stressed. In any case, the minimizing metric can be thought of as the closest one to being flat in a fixed conformal class, since geodesic balls will deviate the least from being Euclidean locally.

Remark 1 Theorem 1 may be suitably modified, so that we are able to get a connection with the prescribed scalar curvature problem. In particular, consider a smooth positive function $r$ on $\bar{M}$. Then, we may ask whether

$$
\inf _{g \in\left[g_{0}\right]}\left\|\frac{R_{g}}{r}\right\|_{L^{\infty}(M)}
$$

is attained. It turns out that if we substitute condition (8) by:

$$
\inf _{u \in A^{\infty}\left(c_{1}, c_{2}, u_{0}\right)}\left\|\frac{R_{g}}{r}\right\|_{L^{\infty}(M)}<\frac{c_{n}}{c_{1}^{2 / n} K_{n}^{2} \sup _{M} r},
$$

then our results are still valid in that context. Thus, in that case the minimizing metric will have prescribed curvature of our choice outside of a set of Lebesgue measure 0 . Nevertheless, for simplicity reasons, we only give a proof in the case $r=1$, leaving the minor modifications in the arguments to the reader.

Notation In what follows, we reserve $C$ for various constants appearing throughout the text. If further clarification is needed on the dependence of the constants on various quantities, we state it explicitly.

\section{A priori bounds}

\subsection{Lower bounds}

We will prove Theorem 1 using an approximation scheme. In particular, since the space $L^{\infty}\left(M, \mu_{0}\right)$ is not reflexive, the Direct Method cannot be used to prove existence of a minimizer. Nevertheless, we first establish existence of minimizers of:

$$
\left(\int_{M} \frac{1}{\mu(M)}|R|^{p} u^{2^{*}} \mathrm{~d} \mu_{0}\right)^{1 / p}
$$

and then pass to the limit as $p \rightarrow \infty$.

The approximation procedure that we follow, makes the existence of a-priori bounds for solutions of (2) necessary, so that we get a bounded nonzero minimizer in the limit. Note that one has to establish existence of both upper and lower bounds, contrary to the lowerdimensional case in [15]. In that context, due to the exponential conformal factor and the surface geometry, the presence of upper bounds only, was sufficient to pass to the limit. 
To prove existence of a lower bound, we take advantage of our boundary condition (7). Since (8) holds, there are some natural bounds on the $L^{p}$ norms of the curvature functionals after a conformal change, for the class of functions that we study. We exploit those two properties, following an idea suggested by Moser (personal communication), along with some applications of standard elliptic regularity estimates to deduce the following:

Proposition 1 Let $E_{0}, p>0$ and $n \geq 3$, be such that $p>\frac{n}{2}$. Then, there exists a positive constant $C_{2}=C_{2}\left(c_{1}, E_{0}, g_{0}, M, n, p, u_{0}\right)$, such that for every positive solution $u \in A^{p}\left(c_{1}, c_{2}, u_{0}\right)$ of the boundary value problem:

$$
\begin{aligned}
-c_{n} \Delta_{g_{0}} u+R_{0} u & =R u^{2^{*}-1}, & & \text { in } M \\
u & =u_{0} & & \text { on } \partial M,
\end{aligned}
$$

with $E_{p}(u)<E_{0}$, it holds that:

$$
u>C_{2} \text {. }
$$

Proof We note that since $u \in A^{p}\left(c_{1}, c_{2}, u_{0}\right)$ and $p>\frac{n}{2}$, the Sobolev Embedding Theorem implies that $u \in C^{0}\left(\bar{M}, g_{0}\right)$. Thus, the set

$$
\Omega=\left\{x \in M \mid u(x)<c_{0}\right\}
$$

is open, for a fixed $c_{0}>0$, independent of $u$ and chosen sufficiently small later on, such that:

$$
\min _{\partial M} u_{0}>c_{0} .
$$

If $\Omega=\emptyset$, there is nothing to prove, hence we assume that $\Omega \neq \emptyset$ from now on. Moreover, we set $\Omega^{-}=\{x \in \Omega \mid R(x)<0\}$. We will estimate $u$ from below in $\Omega$, by suitably constructed solutions of certain equations.

Let $v$ a solution of:

$$
\begin{aligned}
& -c_{n} \Delta_{g_{0}} v+R_{0}^{+} v= \begin{cases}R u^{2^{*}-1}, & \text { in } \Omega^{-}, \\
0, & \text { in } M \backslash \Omega^{-},\end{cases} \\
& v=c_{0} \quad \text { on } \partial M,
\end{aligned}
$$

where $R_{0}^{+}$stands for $R_{0}^{+}=\max \left\{R_{0}, 0\right\}$ as usual. The standard $L^{p}$ theory for elliptic equations (see [7] for example), implies that $v \in W^{2, p}\left(M, g_{0}\right)$ exists. Furthermore, $v \leq c_{0}$ in $\bar{M}$, from the maximum principle and $v \leq u$ in $\bar{M}$, by the comparison principle.

Now, let $w$ a solution of:

$$
\begin{gathered}
c_{n} \Delta_{g_{0}} w-R_{0}^{+} w=0 \text { in } M, \\
w=c_{0} \text { on } \partial M .
\end{gathered}
$$

Then, there exists a $k>0$, independent of the boundary data, such that $w>k c_{0}$ in $M$. In addition, $\bar{v}:=v-w$ is a solution of the problem:

$$
\begin{aligned}
& c_{n} \Delta_{g_{0}} \bar{v}-R_{0}^{+} \bar{v}= \begin{cases}-R u^{2^{*}-1} & \text { in } \Omega^{-}, \\
0, & \text { in } M \backslash \Omega^{-},\end{cases} \\
& \bar{v}=0 \text { on } \partial M .
\end{aligned}
$$


The standard $L^{p}$ regularity theory implies that $\bar{v} \in W_{0}^{1, p}\left(M, g_{0}\right) \cap W^{2, p}\left(M, g_{0}\right)$, with an estimate of the form:

$$
\int_{M}\left|\Delta_{g_{0}} \bar{v}-\frac{1}{c_{n}} R_{0}^{+} \bar{v}\right|^{p} \mathrm{~d} \mu_{0} \leq C c_{0}^{p\left(2^{*}-1\right)} \int_{M}|R|^{p} \mathrm{~d} \mu_{0},
$$

holding, where $C=C(n, p)$. Using the $L^{p}$ theory for solutions of elliptic equations once more, we get an estimate for $\bar{v}$ in $W^{2, p}\left(M, g_{0}\right)$, which, combined with (12), yields the following inequalities:

$$
\left(\|\bar{v}\|_{W^{2, p}\left(M, g_{0}\right)}\right)^{p} \leq C \int_{M}\left|\Delta_{g_{0}} \bar{v}-\frac{1}{c_{n}} R_{0}^{+} \bar{v}\right|^{p} \mathrm{~d} \mu_{0} \leq C c_{0}^{p\left(2^{*}-1\right)} \int_{M}|R|^{p} \mathrm{~d} \mu_{0} .
$$

We then extend this estimate, for $\bar{v}$ in $C^{1}\left(M, g_{0}\right)$, using the Sobolev Embedding Theorem, and get

$$
|v-w| \leq C|| R \|_{L^{p}\left(M, \mu_{0}\right)} c_{0}^{\left(2^{*}-1\right)},
$$

where $C=C(M, n, p)$, after taking into account the definition of $\bar{v}$.

Let $\gamma=\inf _{x \in M} u$, with $\gamma>0$, since $u$ is positive and continuous on $\bar{M}$. Then, using the definition of $E_{p}$, and the fact that $u \in A^{p}\left(c_{1}, c_{2}, u_{0}\right)$, we have:

$$
\|R\|_{L^{p}\left(M, \mu_{0}\right)} \leq\left(\frac{1}{\gamma^{2^{*}}} \int_{M}|R|^{p} u^{2^{*}} \mathrm{~d} \mu_{0}\right)^{1 / p}=\gamma^{-2^{*} / p} c_{1}^{1 / p} E_{p}(u) \leq \gamma^{-2^{*} / p} c_{1}^{1 / p} E_{0} .
$$

Hence, it follows that:

$$
c_{0}\left(k-C c_{0}^{2^{*}-2} c_{1}^{1 / p} E_{0} \gamma^{-2^{*} / p}\right)<w-C\|R\|_{L^{p}\left(M, \mu_{0}\right)} c_{0}^{\left(2^{*}-1\right)}<v \leq \gamma,
$$

since $u \geq v$. Setting $q=\frac{1}{2}+\frac{n}{4 p}$, note that the following relation holds:

$$
\frac{n}{2 p}<q<1
$$

since $p>n / 2$. Moreover,

$$
\left(2^{*}-2\right) q-\frac{2^{*}}{p}=\frac{2-\frac{n}{p}}{n-2}>0 .
$$

If $\gamma>\left(\inf _{\partial M} u_{0}\right)^{1 / q}$, we have a uniform lower bound for $u$ in $\bar{M}$, and there is nothing to prove. So, suppose that $\gamma \leq\left(\inf _{\partial M} u_{0}\right)^{1 / q}$ and set $c_{0}=\gamma^{q}$. Then, Eq. (13) implies that:

$$
\gamma^{1-q} \geq\left(k-C c_{1}^{1 / p} E_{0} \gamma^{\frac{2-\frac{n}{p}}{n-2}}\right) \text {. }
$$

Clearly, a positive lower bound $C_{2}$ for $u$ follows, as long as:

$$
\gamma \geq\left(\frac{k}{2 C c_{1}^{1 / p} E_{0}}\right)^{\frac{n-2}{2-\frac{n}{p}}}
$$

Otherwise, the last inequality implies:

$$
\gamma \geq\left(\frac{k}{2}\right)^{\frac{1}{1-q}}
$$

allowing us to finish the proof in that case as well. 


\subsection{Upper bounds}

Now that we have established existence of lower bounds for solutions of (2), it remains to prove that uniform upper bounds exist too. We begin with the statement of the:

Theorem 2 Let $u \in A^{p_{0}}\left(c_{1}, c_{2}, u_{0}\right)$ be a positive solution of the equation:

$$
-c_{n} \Delta_{g_{0}} u+R_{0} u=R u^{2^{*}-1},
$$

for $p_{0}>n$ fixed. In addition, let $\delta$ such that:

$$
0<\delta<\frac{c_{n}}{K_{n}^{2}},
$$

where $K_{n}$ is the best constant for the Euclidean Sobolev Inequality. Then, if the following statement is true:

$$
c_{1}^{\frac{2 p_{0}}{n}-1} \int_{M}|R|^{p_{0}} u^{2^{*}} \mathrm{~d} \mu_{0} \leq \delta^{p_{0}},
$$

there exists a positive constant $C=C\left(M, \delta, n, p_{0}, c_{1}, c_{2}, g_{0}, u_{0}\right)$, such that:

$$
u(x) \leq C,
$$

$\forall x \in \bar{M}$

The proof of Theorem 2 consists of a blow-up type argument used widely in that context (see [2] for a detailed survey in the case of closed manifolds). In particular, we argue indirectly, supposing that no uniform upper bounds exist for a sequence $u_{\alpha}$ of solutions to (14). Then, the exponential map can be used to transfer our sequence of solutions to $\mathbb{R}^{n}$. In the case the boundary is not involved, the sharp Euclidean Sobolev inequality and (16) allow us to reach a contradiction. If that is not the case, some subtleties arise, which we overcome using standard techniques, similar to the ones in [8].

Proof To reach a contradiction, suppose that condition (17) does not hold. Then, we can choose a sequence of solutions $\left\{u_{\alpha}\right\}_{\alpha \in \mathbb{N}}$ for (14), which satisfy (16), such that:

$$
\sup _{x \in M} u_{\alpha}(x) \rightarrow \infty
$$

as $\alpha \rightarrow \infty$.

Nevertheless, note that multiplying Eq. (14) by $u_{\alpha}$, integrating by parts and using the triangle inequality yields:

$$
\left.\left|c_{n} \int_{M}\right| \nabla u_{\alpha}\right|^{2} \mathrm{~d} \mu_{0}-c_{n} \int_{\partial M} u_{\alpha} \frac{\partial u_{\alpha}}{\partial v} \mathrm{~d} \sigma_{0}\left|\leq \int_{M}\right| R_{0}\left|u_{\alpha}^{2} \mathrm{~d} \mu_{0}+\int_{M}\right| R_{\alpha} \mid u_{\alpha}^{2^{*}} \mathrm{~d} \mu_{0} .
$$

Then, constraint (4), Eq. (16) and Holder's inequality, imply that the terms in the righthandside are uniformly bounded. Moreover, the boundary term satisfies

$$
\left|\int_{\partial M} u_{\alpha} \frac{\partial u_{\alpha}}{\partial v} \mathrm{~d} \sigma_{0}\right| \leq C
$$

for a constant $C$ independent of $\alpha$, as is evident after using the triangle inequality, Holder's inequality and the boundary conditions (6), (7). Hence, using Eqs. (19) and (20), we deduce that $\int_{M}\left|\nabla u_{\alpha}\right|^{2} \mathrm{~d} \mu_{0} \leq C$, with $C$ independent of $\alpha$, which in terms implies that $u_{\alpha}$ is uniformly bounded in $W^{1,2}\left(M, g_{0}\right)$. Any constant appearing from now on, should be assumed independent of $\alpha$, unless otherwise noted. 
Let $x_{\alpha} \in \bar{M}$ such that $u_{\alpha}\left(x_{\alpha}\right)=\sup _{x \in M} u_{\alpha}(x)$. Also, let $\lambda_{\alpha}^{\frac{2-n}{2}}=u_{\alpha}\left(x_{\alpha}\right)$, and note that $\lambda_{\alpha} \rightarrow 0$ by our assumptions on $u_{\alpha}$. Moreover, consider the following quantity:

$$
d_{\alpha}=\frac{\operatorname{dist}_{g_{0}}\left(x_{\alpha}, \partial M\right)}{\lambda_{\alpha}} .
$$

Then we can assume that, up to choosing a subsequence, $d_{\alpha} \rightarrow d_{0}$, for some $d_{0} \in[0, \infty]$. We will distinguish two cases in what follows, depending on the values of $d_{0}$.

Case 1 We first consider the case $d_{0}=\infty$. Note that by compactness, there exists a point $x_{0} \in \bar{M}$ such that, up to selecting a subsequence, we have:

$$
x_{\alpha} \rightarrow x_{0},
$$

as $\alpha \rightarrow \infty$. Let $\hat{u}_{\alpha}$ the sequence of functions:

$$
\hat{u}_{\alpha}(x)=\lambda_{\alpha}^{\frac{n-2}{2}} u_{\alpha}\left(\exp _{x_{\alpha}}\left(\lambda_{\alpha} x\right)\right)
$$

where $\exp _{x_{\alpha}}$ stands for the exponential map at $x_{\alpha}$. Then, for $\alpha$ large enough, $\hat{u}_{\alpha}$ is well defined in a ball $B_{R}(0)$ of radius $R>0$ around 0 , since $d_{0}=\infty$. In addition, it holds that $0 \leq \hat{u}_{\alpha} \leq 1$, with $\hat{u}_{\alpha}(0)=1$, as is evident from the definition of the sequence and standard properties of the exponential map. Finally, the change of coordinates we are using, yields induced metrics $\hat{g}_{\alpha}(x)=\exp _{x_{\alpha}}^{*} g_{0}\left(\lambda_{\alpha} x\right)$, with corresponding measures $\hat{\mu}_{\alpha}$, gradients $\hat{\nabla}^{\alpha}$ and Laplace-Beltrami operators $\Delta_{\hat{g}_{\alpha}}$. In particular,

$$
\hat{g}_{\alpha} \rightarrow g_{\text {euc }}
$$

holds locally, where $g_{\text {euc }}$ stands for the standard metric in $\mathbb{R}^{n}$.

Consider the rescaled functions given by $\hat{R}_{0 \alpha}=\lambda_{\alpha}^{2} R_{0}\left(\exp _{x_{\alpha}}\left(\lambda_{\alpha} x\right)\right)$ and $\hat{R}_{\alpha}=$ $R_{\alpha}\left(\exp _{x_{\alpha}}\left(\lambda_{\alpha} x\right)\right)$, which correspond to $R_{0}$ and $R_{\alpha}$ respectively, in our new coordinates. Then, the sequence $\hat{u}_{\alpha}$ satisfies the equation:

$$
-c_{n} \Delta_{\hat{g}_{\alpha}} \hat{u}_{\alpha}+\hat{R}_{0 \alpha} \hat{u}_{\alpha}=\hat{R}_{\alpha} \hat{u}_{\alpha}^{2^{*}-1}
$$

in $B_{R}(0)$, for every $R>0$, as long as $\alpha$ is large enough. Moreover, the following estimates hold

$$
\begin{gathered}
\int_{B_{R}(0)}\left|\hat{\nabla}^{\alpha} \hat{u}_{\alpha}\right|^{2} \mathrm{~d} \hat{\mu}_{\alpha} \leq C, \\
\int_{B_{R}(0)} \hat{u}_{\alpha}^{2^{*}} \mathrm{~d} \hat{\mu}_{\alpha} \leq C,
\end{gathered}
$$

for every radius $R>0$ and for $\alpha$ large enough, after using the change of variables formula.

We now proceed using a cut-off function argument as in [2], for a smooth function $0 \leq$ $\eta \leq 1$, with:

$$
\eta(x)= \begin{cases}1 & \text { in } B_{R / 2}(0) \\ 0 & \text { in } \mathbb{R}^{n} \backslash B_{3 R / 4}(0),\end{cases}
$$

for some $R$ independent of $\alpha$. Letting $\eta_{\alpha}(x)=\eta\left(\lambda_{\alpha} x\right)$, we note that by the definition of $\eta$ we have:

$$
\left|\hat{\nabla}^{\alpha} \eta_{\alpha}\right| \leq C \lambda_{\alpha}
$$

Following that, we derive some more estimates on $\eta_{\alpha} \hat{u}_{\alpha}$ to establish some regularity results. Trivially, it holds that:

$$
\int_{\mathbb{R}^{n}}\left|\hat{\nabla}^{\alpha}\left(\eta_{\alpha} \hat{u}_{\alpha}\right)\right|^{2} \mathrm{~d} \hat{\mu}_{\alpha} \leq 2\left(\int_{\mathbb{R}^{n}}\left|\hat{\nabla}^{\alpha} \eta_{\alpha}\right|^{2}\left|\hat{u}_{\alpha}\right|^{2} \mathrm{~d} \hat{\mu}_{\alpha}+\int_{\mathbb{R}^{n}}\left|\hat{\nabla}^{\alpha} \hat{u}_{\alpha}\right|^{2}\left|\eta_{\alpha}\right|^{2} \mathrm{~d} \hat{\mu}_{\alpha}\right) .
$$


Proceeding to a closer examination of the terms in (27), we obtain the following estimate:

$$
\int_{\mathbb{R}^{n}}\left|\hat{\nabla}^{\alpha} \hat{u}_{\alpha}\right|^{2}\left|\eta_{\alpha}\right|^{2} \mathrm{~d} \hat{\mu}_{\alpha} \leq \int_{B_{R}(0)}\left|\hat{\nabla}^{\alpha} \hat{u}_{\alpha}\right|^{2} \mathrm{~d} \hat{\mu}_{\alpha} \leq C,
$$

for some $R>0$, using (23). Moreover, if we turn our attention to the remaining term, we have:

$$
\int_{\mathbb{R}^{n}}\left|\hat{\nabla}^{\alpha} \eta_{\alpha}\right|^{2}\left|\hat{u}_{\alpha}\right|^{2} \mathrm{~d} \hat{\mu}_{\alpha} \leq\left(\int_{\mathbb{R}^{n}}\left|\hat{\nabla}^{\alpha} \eta_{\alpha}\right|^{n} \mathrm{~d} \hat{\mu}_{\alpha}\right)^{2 / n}\left(\int_{B_{3} / 4 \lambda_{\alpha}(0)} \hat{u}_{\alpha}^{2^{*}} \mathrm{~d} \hat{\mu}_{\alpha}\right)^{2 / 2^{*}} \leq C,
$$

using (24), (26) and Holder's inequality. Thus the last two estimates imply that $\int_{\mathbb{R}^{n}}\left|\hat{\nabla}^{\alpha}\left(\eta_{\alpha} \hat{u}_{\alpha}\right)\right|^{2} \mathrm{~d} \hat{\mu}_{\alpha} \leq C$. Combing this estimate and (24), it follows that up to a subsequence $\eta_{\alpha} \hat{u}_{\alpha} \rightarrow \hat{u}$, weakly in the homogeneous Sobolev space $D_{1}^{2}\left(\mathbb{R}^{n}, \mu_{\text {euc }}\right)$ and strongly in $L^{2^{*}}\left(\mathbb{R}^{n}, \mu_{\text {euc }}\right)$, for a limit function $\hat{u}$. Hence:

$$
\|\hat{u}\|_{L^{2^{*}}\left(B_{R}(0), \mu_{\mathrm{euc}}\right)} \leq \liminf _{\alpha \rightarrow+\infty}\left\|\eta_{\alpha} \hat{u}_{\alpha}\right\|_{L^{2^{*}\left(B_{R}(0), \hat{\mu}_{\alpha}\right)}} \leq C
$$

holds, for every $R>0$, following the weak convergence, Eqs. (21), (24) and the fact that $\eta \leq 1$. Note that the constant $C$ is independent of $R$.

It remains to prove that $\hat{u} \neq 0$. We will do so, using the Harnack inequality Lemma 3.4 from [2], based on an inequality in [9]. In that direction, let $f_{\alpha}:=\left(\hat{R}_{\alpha} \hat{u}_{\alpha}^{2^{*}-2}-\hat{R}_{0 \alpha}\right)$. Then, the following inequalities:

$$
\int_{B_{R}(0)}\left|\hat{R}_{\alpha}\right|^{n / 2} \hat{u}_{\alpha}^{2^{*}} \mathrm{~d} \hat{\mu}_{\alpha} \leq c_{1}^{1-\frac{n}{2 p_{0}}}\left(\int_{M}\left|R_{\alpha}\right|^{p_{0}} u_{\alpha}^{2^{*}} \mathrm{~d} \mu_{0}\right)^{\frac{n}{2 p_{0}}} \leq \delta^{n / 2},
$$

are valid $\forall R>0$, after using (16). In addition, Holder's inequality and the fact that $\hat{u}_{\alpha} \leq 1$, imply a bound:

$$
\int_{B_{1}(0)}\left|f_{\alpha}\right|^{s} \mathrm{~d} \hat{\mu}_{\alpha} \leq C
$$

for $s \leq p_{0}$. Using the aforementioned Harnack inequality, we derive:

$$
1=\sup _{B_{1 / 2}(0)} \hat{u}_{\alpha} \leq C\left\|\hat{u}_{\alpha}\right\|_{L^{1}\left(B_{1}(0), \hat{\mu}_{\alpha}\right)} .
$$

It then follows that $\hat{u} \neq 0$, since $\hat{\eta}_{\alpha} \hat{u}_{\alpha}=\hat{u}_{\alpha} \rightarrow \hat{u}$, strongly in $L^{1}\left(B_{1}(0)\right)$, as $\alpha \rightarrow \infty$.

Turning our attention to $\hat{R}_{\alpha}$, we have:

$$
\hat{R}_{\alpha} \hat{u}_{\alpha}^{2^{*}-2} \rightarrow f \text { in } L^{\frac{n}{2}}\left(\mathbb{R}^{n}, \mu_{\mathrm{euc}}\right),
$$

for a limit function $f$, up to a subsequence. Moreover, by the definition of $\hat{R}_{0 \alpha}$, and since $\hat{u}_{\alpha} \leq 1$, it holds that:

$$
\left|\int_{B_{R}(0)} \hat{R}_{0 \alpha} \hat{u}_{\alpha} \eta_{\alpha} \phi \mathrm{d} \hat{\mu}_{\alpha}\right| \leq C \max _{M}\left|R_{0}\right| \lambda_{\alpha}^{2}
$$

for every $\phi \in C_{0}^{\infty}\left(\mathbb{R}^{n}\right)$. Note that the righthandside goes to 0 as $\alpha \rightarrow+\infty$. Similarly, after using Eq. (26), we have

$$
\left|\int_{B_{R}(0)}\left(\hat{\nabla}^{\alpha} \hat{u}_{\alpha} \hat{\nabla}^{\alpha} \eta_{\alpha} \phi\right) \mathrm{d} \hat{\mu}_{\alpha}\right| \leq C \lambda_{\alpha} \int_{B_{R}(0)}\left|\left(\hat{\nabla}^{\alpha} \hat{u}_{\alpha}\right) \phi\right| \mathrm{d} \hat{\mu}_{\alpha},
$$


for $\phi \in C_{0}^{\infty}\left(\mathbb{R}^{n}\right)$, with the latter expression tending to 0 when $\alpha \rightarrow \infty$ as well. Then, (22) implies

$$
c_{n} \int_{B_{R}(0)}\left(\nabla^{E u} \hat{u}_{\alpha} \nabla^{E u} \phi\right) \eta_{\alpha} \mathrm{d} \mu_{\mathrm{euc}}=\int_{B_{R}(0)} \hat{R}_{\alpha} \hat{u}_{\alpha}^{2^{*}-1} \phi \mathrm{d} \mu_{\mathrm{euc}}+o(1),
$$

for every $\phi \in C_{0}^{\infty}\left(\mathbb{R}^{n}\right)$ and $\alpha$ large enough, after multiplying by $\eta_{\alpha} \phi$ and integrating by parts. Here $\nabla^{E u}$ stands for the ordinary gradient in $\mathbb{R}^{n}$. Using the various convergence modes that we have established, we pass to the limit as $\alpha \rightarrow+\infty$ and get:

$$
c_{n} \int_{\mathbb{R}^{n}}\left(\nabla^{E u} \hat{u} \nabla^{E u} \phi\right) \mathrm{d} \mu_{\mathrm{euc}} \leq \int_{\mathbb{R}^{n}} f \hat{u} \phi \mathrm{d} \mu_{\mathrm{euc}},
$$

for every $\phi \in C_{0}^{\infty}\left(\mathbb{R}^{n}\right)$. Inserting $\phi=\hat{u}$ and using Holder's inequality, we have

$$
c_{n} \int_{\mathbb{R}^{n}}\left|\nabla^{E u} \hat{u}\right|^{2} \mathrm{~d} \mu_{\text {euc }} \leq\left(\int_{\mathbb{R}^{n}}|f|^{n / 2} \mathrm{~d} \mu_{\text {euc }}\right)^{2 / n}\left(\int_{\mathbb{R}^{n}} \hat{u}^{2^{*}} \mathrm{~d} \mu_{\text {euc }}\right)^{2 / 2^{*}} .
$$

But from the definition of weak convergence and (28) it follows that:

$$
\left(\int_{\mathbb{R}^{n}}|f|^{\frac{n}{2}} \mathrm{~d} \mu_{\text {euc }}\right)^{2 / n} \leq \lim _{R \rightarrow \infty} \liminf _{\alpha \rightarrow+\infty}\left\|\hat{R}_{\alpha} \hat{u}_{\alpha}^{2^{*}-2}\right\|_{L^{\frac{n}{2}\left(B_{R}(0), \hat{\mu}_{\alpha}\right)}} \leq \delta .
$$

Thus (15) immediately implies that:

$$
\int_{\mathbb{R}^{n}}\left|\nabla^{E u} \hat{u}\right|^{2} \mathrm{~d} \mu_{\text {euc }}<\frac{1}{K_{n}^{2}}\left(\int_{\mathbb{R}^{n}} \hat{u}^{2^{*}} \mathrm{~d} \mu_{\text {euc }}\right)^{\frac{2}{2^{*}}},
$$

which contradicts the sharp Euclidean Sobolev inequality :

$$
\|\hat{u}\|_{L^{2^{*}\left(\mathbb{R}^{n}\right)}} \leq K_{n}\left\|\nabla^{E u} \hat{u}\right\|_{L^{2}\left(\mathbb{R}^{n}\right)},
$$

proving our argument when $d_{0}=\infty$.

Case 2 In the case $0 \leq d_{0}<\infty$, we infer that up to a subsequence $x_{\alpha} \rightarrow x_{0} \in \partial M$ holds, as $\alpha$ goes to $\infty$. Thus, we need to consider boundary data in that case. Using standard arguments, see [8] for example, we may assume that near $x_{0}$ the boundary of our domain is included in the halfspace $\left\{x_{n}=-d_{\alpha}\right\}$, after using a straightening argument if necessary. Here $x_{n}$ stands for the last coordinate in $\mathbb{R}^{n}$ as usual.

When $d_{\alpha} \neq 0, \hat{u}_{\alpha}$ is well defined in the half-space $B_{R}(0) \cap\left\{x_{n}>-d_{\alpha}\right\}$, as long as $R<d_{0}$. Moreover, we extend $\hat{u}_{\alpha}$ on the boundary using $u_{0}$. Thus

$$
-c_{n} \Delta_{\hat{g}_{\alpha}} \hat{u}_{\alpha}+\hat{R}_{0 \alpha} \hat{u}_{\alpha}=\hat{R}_{\alpha} \hat{u}_{\alpha}^{2^{*}-1}
$$

holds in any ball $B_{R}(0)$, for $\alpha$ sufficiently large, after extending the scalar curvature functions $\hat{R}_{\alpha}$ and $\hat{R}_{0 \alpha}$ by 0 , outside of $\left\{x_{n}>-d_{\alpha}\right\}$.

We may also define $\hat{f}_{\alpha}$ and $\hat{u}$, like in the first part of our proof. Our functions are supported in the halfspace $\left\{x_{n}>-d_{\alpha}\right\}$ in that case, but they remain well defined in any ball around 0 , as long as $\alpha$ is large enough. The Harnack inequality that we used is still valid in our new context too. Since the rest of our arguments from the first part of the proof carry over under those modifications, the sharp Sobolev inequality also yields a contradiction in that case.

In the case that $d_{0}=0, \hat{u}_{\alpha}$ is well defined in a half ball $B_{R}^{+}(0)$ of radius $R>0$, as long as $\alpha$ is large enough. Moreover, $\hat{u}_{\alpha}$ satisfies

$$
-c_{n} \Delta_{\hat{g}_{\alpha}} \hat{u}_{\alpha}=\hat{f}_{\alpha} \hat{u}_{\alpha}, \quad \text { in } B_{R}^{+},
$$


and we may use $u_{0}$ to extend $\hat{u}_{\alpha}$ on the boundary. In particular, when $x \in\left\{x_{n}=0\right\}$

$$
\hat{u}_{\alpha}(x) \rightarrow 0,
$$

as $\alpha \rightarrow \infty$, since $\lambda_{\alpha} \rightarrow 0$ and the boundary data are independent of $\alpha$. Also, we still have $\hat{u}_{\alpha}\left(x_{\alpha}\right)=1$, as is evident from the definition of $\hat{u}_{\alpha}$. Finally, note that

$$
\int_{B_{1}^{+}(0)}\left|\hat{f}_{\alpha}\right|^{s} \mathrm{~d} \hat{\mu}_{\alpha} \leq C
$$

holds for a fixed $s$, with $n / 2<s<p_{0}$.

The coefficients of $\Delta_{\hat{g}_{\alpha}}$ converge smoothly to those of the usual Euclidean Laplacian, as $\alpha \rightarrow 0$. This fact along with the smooth boundary data on $\partial B_{R}^{+}$allow us to use the standard elliptic regularity theory in $B_{1}^{+}(0)$. Hence, we first obtain a uniform bound for $\hat{u}_{\alpha}$ in $W^{2, p}\left(B_{1}^{+}(0)\right)$, for $p>n / 2$, after taking into account Eq. (33). The Sobolev Embedding Theorem then implies that:

$$
\left\|\hat{u}_{\alpha}\right\|_{C^{0, \gamma}\left(B_{1}^{+}(0), \hat{g}_{\alpha}\right)} \leq C,
$$

for some $0<\gamma<1$. Thus our sequence $\hat{u}_{\alpha}$ is equicontinuous. Moreover, since $\hat{u}_{\alpha} \leq 1$ holds, we may infer that a function $\tilde{u}$ exists, such that:

$$
u_{\alpha} \rightarrow \tilde{u},
$$

uniformly as $\alpha \rightarrow \infty$, by means of the Arzela-Ascoli Theorem. Hence, up to selecting a subsequence, we have:

$$
u_{\alpha}\left(x_{\alpha}\right) \rightarrow \tilde{u}(0)=1,
$$

uniformly as $\alpha \rightarrow \infty$. The latter clearly contradicts (32), thus concluding our proof in that case too.

\section{Existence and uniform estimates for the $p$-problem}

The presence of uniform a-priori bounds, allows us to use the Direct Method to establish existence of minimizers for the approximating functionals $E_{p}(u)=\left(\int_{M} \frac{1}{\operatorname{vol}(M, \mu)}|R|^{p} u^{2^{*}} \mathrm{~d} \mu_{0}\right)^{1 / p}$. In particular, we have the following:

Proposition 2 Let $c_{1}>0, u_{0} \in C^{\infty}(\bar{M})$ be given. Then $\forall \delta>0$ and for every fixed $p_{0}$ with $p_{0}>n$, such that:

$$
\delta<\frac{c_{n}}{K_{n}^{2}}
$$

and

$$
\inf _{u \in A^{p_{0}}\left(c_{1}, c_{2}, u_{0}\right)} E_{p_{0}}(u)<c_{1}^{1 / p_{0}-2 / n} \delta,
$$

there exists a minimizer $u_{p_{0}}$ of the functional $E_{p_{0}}(u)$ in the set $A^{p_{0}}\left(c_{1}, c_{2}, u_{0}\right)$.

Proof Let $\left\{u_{k}\right\}_{k \in \mathbb{N}} \subset A^{p_{0}}\left(c_{1}, c_{2}, u_{0}\right)$ a minimizing sequence for $E_{p_{0}}$, with corresponding scalar curvature $R_{k}$. Then for $p_{0}>n$ and $k$ large enough,

$$
\int_{M}\left|R_{k}\right|^{p_{0}} u_{k}^{2^{*}} \mathrm{~d} \mu_{0}<\delta^{p_{0}} c_{1}^{1-2 p_{0} / n}
$$


holds, and there exist positive constants $C_{1}$ and $C_{2}$ independent of $k$, such that $C_{2} \leq u_{k} \leq C_{1}$ in $\bar{M}$, following Proposition 1 and Theorem 2. Thus, standard elliptic estimates imply that:

$$
\left\|u_{k}\right\|_{W^{2, p_{0}\left(M, g_{0}\right)}} \leq C\left[\left\|u_{k}\right\|_{L^{p_{0}\left(M, \mu_{0}\right)}}+\left\|R_{k} u_{k}^{\frac{2^{*}}{p_{0}}}\right\|_{L^{p_{0}\left(M, \mu_{0}\right)}}+\left\|u_{0}\right\|_{W^{2, p_{0}\left(M, g_{0}\right)}}\right] \leq C,
$$

for a constant $C$ independent of $k$. In addition, there exists a limit function $u \neq 0$, in light of Proposition 1, such that:

$$
u_{k} \rightarrow u,
$$

weakly in $W^{2, p_{0}}\left(M, g_{0}\right)$ and strongly in $C^{1}\left(\bar{M}, g_{0}\right)$. Hence, a uniform bound in $C^{1, \alpha}\left(M, g_{0}\right)$ follows, for $\alpha \in\left(0,1-\frac{n}{p_{0}}\right)$ via the Sobolev Embedding Theorem. On the other hand, we also have $R_{k} \rightarrow R$ weakly in $L^{q}\left(M, \mu_{0}\right)$, up to a subsequence, for a limit curvature $R$ and every $q<\infty$. Then, integration by parts and the Dominated Convergence Theorem yield:

$$
\int_{M} R_{k} u_{k}^{2^{*}-1} \phi \mathrm{d} \mu_{0}=\lim _{k \rightarrow \infty}\left[-c_{n} \int_{M} u_{k} \Delta_{g_{0}} \phi \mathrm{d} \mu_{0}+\int_{M} R_{0} u_{k} \phi \mathrm{d} \mu_{0}\right]=\int_{M} R u^{2^{*}-1} \phi \mathrm{d} \mu_{0},
$$

for $\phi \in C_{0}^{\infty}\left(M, g_{0}\right)$, proving that $R$ is the curvature of $u$.

The lower semicontinuity of the $L^{p_{0}}$ norm in $M$ with respect to $\mu_{0}$, implies that the infimum of $E_{p_{0}}$ is achieved, using the lower semicontinuity of the functional with respect to weak convergence. Hence, it remains to prove that $u \in A^{p_{0}}\left(c_{1}, c_{2}, u_{0}\right)$. Indeed, Theorem 2 and the $C^{1}$-convergence we established, imply that the volume constraint is preserved. Finally, the constraint (6) is preserved too, over the boundary, since the $C^{1}$ convergence holds in all of $\bar{M}$, thus concluding the proof.

Note that for the minimization of $E_{p}(u)$ under constraints (4), (6) and (7), there exist Lagrange multipliers $a_{p}, b_{p} \in \mathbb{R}$. If $u_{p}$ is a minimizer satisfying those constraints, and $R_{p}$ is the scalar curvature of the metric $g_{p}=u_{p}^{2^{*}-2} g_{0}$, the Euler-Lagrange equations for our problem are as follows:

$$
\begin{gathered}
-\Delta_{g_{p}}\left(\left|R_{p}\right|^{p-2} R_{p}\right)+\left[\frac{n}{2 p(n-1)}-\frac{1}{n-1}\right]\left|R_{p}\right|^{p}=a_{p}, \quad \text { on } M, \\
\left|R_{p}\right|^{p-2} R_{p}=b_{p}, \quad \text { on } \partial M .
\end{gathered}
$$

We remark that $\frac{n}{2 p(n-1)}-\frac{1}{n-1} \neq 0$ under our assumptions on $p$. Let

$$
\gamma_{p}=\max \left\{\left|a_{p}\right|,\left|b_{p}\right|,|| R_{p} \|_{L^{p}\left(\mu_{p}\right)}^{p-1}\right\}
$$

and consider the quantities $\alpha_{p}=a_{p} / \gamma_{p}, \beta_{p}=b_{p} / \gamma_{p}$ and $w_{p}=\frac{\left|R_{p}\right|^{p-2} R_{p}}{\gamma_{p}}$. It follows that the initial fourth order boundary value problem can now be reformulated, as a second order one:

$$
\begin{gathered}
-\Delta_{g_{p}} w_{p}+\left[\frac{n}{2 p(n-1)}-\frac{1}{n-1}\right] R_{p} w_{p}=\alpha_{p}, \quad \text { on } M, \\
w_{p}=\beta_{p}, \quad \text { on } \partial M,
\end{gathered}
$$

with $\alpha_{p}, \beta_{p} \in[-1,1]$. 
Equation (8) is satisfied, so it holds that:

$$
\limsup _{p \rightarrow \infty}\left\|R_{p}\right\|_{L^{p}\left(M, \mu_{p}\right)}<\frac{c_{n}}{c_{1}^{2 / n} K_{n}^{2}} .
$$

Also, from the definition of $w_{p}$, it follows that:

$$
\left\|w_{p}\right\|_{L^{p^{\prime}}\left(M, \mu_{p}\right)} \leq 1,
$$

after using the renormalization (36). Since $R_{p}$ is uniformly bounded in $L^{p}\left(M, \mu_{p}\right)$ and $w_{p}$ is uniformly bounded in $L^{p^{\prime}}\left(M, \mu_{p}\right)$, we conclude, using Holder's inequality, that the term:

$$
k_{p}:=\alpha_{p}-\left[\frac{n}{2 p(n-1)}-\frac{1}{n-1}\right] R_{p} w_{p},
$$

is uniformly bounded in $L^{1}\left(M, \mu_{0}\right)$.

Note that we may interpret $k_{p} \mu_{0}$ as a bounded sequence of Radon measures $\mu_{k_{p}}$. A standard compactness result in that case (Theorem 1.3.2 in [6]), leads us to the conclusion that $w_{p} \in W^{1, q}\left(M, g_{0}\right)$, for every $1 \leq q<\frac{n}{n-1}$. Moreover, the uniform $L^{1}$ boundedness of $R_{p} w_{p}$ implies that $w_{p}$ is uniformly bounded in $W^{1, q}\left(M, g_{0}\right)$ too, for $1 \leq q<\frac{n}{n-1}$. Then, the Sobolev Embedding Theorem allows us to deduce that $w_{p}$ is uniformly bounded in $L^{\tilde{p}}\left(M, \mu_{0}\right)$, for every $\tilde{p}=\frac{n q}{n-q}$, with $q<\frac{n}{n-1}$. This in turn implies that $w_{p}$ is uniformly bounded in $L^{\tilde{p}}\left(M, \mu_{0}\right)$, for every $\tilde{p}<\frac{n}{n-2}$. In addition, a direct application of Holder's inequality implies that $R_{p} w_{p}$ is uniformly bounded in $L^{\lambda}\left(M, \mu_{0}\right)$, for every $\lambda<n /(n-2)$ and for $p$ large enough. Furthermore, we may use the standard elliptic $L^{p}$ theory, since the leading order coefficients of $\Delta_{g_{p}}$ are uniformly bounded in $C^{1, \alpha}\left(M, g_{0}\right)$, for some $\alpha \in(0,1)$. Thus, $w_{p}$ is uniformly bounded in $W^{2, q}\left(M, g_{0}\right)$, for every $q<\frac{n}{n-2}$.

To obtain further regularity results bootstrapping is needed, hence we have the following:

Lemma 1 The sequence $w_{p}$, defined as above, is uniformly bounded in $C^{0, \alpha}\left(M, g_{0}\right), \forall a<1$ and converges up to a subsequence, uniformly to a limit function $w$.

Proof We first claim that $w_{p}$ is uniformly bounded in $L^{q}\left(M, \mu_{0}\right)$, for every $q<\frac{n}{n-l}$, provided that $2 \leq l<n$. We will prove our claim using induction in $l$. In the base case $l=2$, the Sobolev Embedding Theorem guarantees that $w_{p}$ is uniformly bounded in $L^{q}\left(M, \mu_{0}\right)$, for every $q<\frac{n}{n-2}$. Now, suppose that for $l_{0} \in \mathbb{N}$, we have $w_{p}$ uniformly bounded in $L^{q}\left(M, \mu_{0}\right)$ with

$$
q<\frac{n}{n-l_{0}} .
$$

For the induction step, suppose that:

$$
l_{0}+1<n \text {. }
$$

From our inductive hypothesis, we get a uniform bound for $w_{p}$ in $L^{q}\left(M, \mu_{0}\right)$, for every $q<$ $\frac{n}{n-l_{0}}$. Then, a uniform bound for $R_{p} w_{p}$ in $L^{\tilde{q}}\left(M, \mu_{0}\right)$ follows using Holder's inequality, when $\tilde{q}<\frac{n}{n-l_{0}}$. Moreover, the partial differential equation for $w_{p}$, combined with the standard elliptic regularity theory, provide us with a uniform bound for $w_{p}$ in $W^{2, \tilde{q}}\left(M, g_{0}\right)$, when $\tilde{q}<\frac{n}{n-l_{0}}$ again. Finally, the Sobolev Embedding Theorem implies a uniform bound for every

$$
q<\frac{n}{n-\left(l_{0}+2\right)}
$$

proving our claim. 
It follows that we can always achieve uniform $L^{\tilde{q}}$ bounds, for $\frac{n}{2}<\tilde{q}$, by letting $l \in \mathbb{N}$, be sufficiently large if necessary. A standard application of standard elliptic estimates provides us with uniform bounds in $W^{2, \tilde{q}}\left(M, g_{0}\right)$. Hence, $w_{p_{k}}$ is a uniformly bounded sequence in $C^{0, \alpha}\left(M, g_{0}\right), \forall \alpha<1$, which is equicontinuous. Thus, the Arzela-Ascoli Theorem applies, and there exists a subsequence of $w_{p}$, which we still call $w_{p}$, which converges uniformly to a limit $w$, concluding our proof.

\section{Proof of the main result}

Up to now, we have proved existence of a positive minimizer $u_{p}$ of $E_{p}$, for every $p \in(n, \infty)$. Moreover, by the regularity results we have obtained, there exists a subsequence $p_{k} \rightarrow \infty$, such that:

1. $u_{p_{k}} \rightarrow u$ in $W^{2, q}\left(M, g_{0}\right)$ and $u_{p_{k}} \rightarrow u$ in $C^{1}\left(\bar{M}, g_{0}\right)$,

2. $w_{p_{k}} \rightarrow w$ uniformly,

3. $R_{p_{k}} \rightarrow \hat{R}$ in $L^{q}\left(M, \mu_{0}\right)$, for a limit curvature $\hat{R}$ and for every $q<\infty$.

Then $R_{p_{k}}=u_{p_{k}}^{1-2^{*}}\left[-c_{n} \Delta_{g_{0}} u_{p_{k}}+R_{0} u_{p_{k}}\right] \rightarrow \hat{R}$, so we deduce that:

$$
\hat{R}=R=u^{1-2^{*}}\left[-c_{n} \Delta_{g_{0}} u+R_{0} u\right] .
$$

In addition, $w$ is a weak solution of the problem

$$
\begin{gathered}
-\Delta_{g} w-\frac{1}{n-1} R w=\alpha, \quad \text { in } M, \\
w=\beta, \quad \text { on } \partial M,
\end{gathered}
$$

following (37), after letting $p \rightarrow \infty$. Here $\alpha, \beta$ are the limits of $\alpha_{p_{k}}, \beta_{p_{k}}$ respectively, and $\Delta_{g}$ is the Laplacian of the metric $g=u^{2^{*}-2} g_{0}$. Moreover, $w$ is not identically zero in $\bar{M}$. Indeed, recalling (36), we remark that if $\alpha \neq 0$, then $w$ is not 0 identically zero in the interior, and if $\beta \neq 0$ then $w \neq 0$ on the boundary. In the remaining case, we have $\left\|w_{p_{k}}\right\|_{L^{p_{k}^{\prime}\left(M, \mu_{p_{k}}\right)}}=1$, for $k$ large enough, and since our convergence is uniform, we are done.

Let $\Gamma=w^{-1}(\{0\})$ and $k \rightarrow \infty$. Then $\log \left|w_{p_{k}}\right|^{\frac{1}{p_{k}-1}} \rightarrow 0$, outside of $\Gamma$, since $w_{p_{k}} \rightarrow w$ uniformly. This in turns implies:

$$
\left|w_{p_{k}}\right|^{\frac{1}{p_{k}-1}} \rightarrow 1
$$

as $k \rightarrow \infty$, locally uniformly in $M \backslash \Gamma$. In addition, up to a subsequence, we have:

$$
\gamma_{p_{k}}^{1 /\left(p_{k}-1\right)} \rightarrow \gamma_{\infty}
$$

as $k \rightarrow \infty$, for some $\gamma_{\infty} \in[0, \infty]$. Hence, from the definition of $w_{p}$, it holds that $|R|=\gamma_{\infty}$ in $M \backslash \Gamma$. Note that the set $\Gamma$ is closed relative to $M$, as the intersection of the closed set $\Gamma$ with $M$. In addition, $R<\infty$ obviously, from Eq. (38). Then, since $w \neq 0$ and $\Gamma \neq M$, we are able to deduce $\gamma_{\infty}<\infty$.

The definition of $u_{p}$ implies that:

$$
E_{p}\left(u_{p}\right) \leq E_{p}\left(u_{q}\right)
$$

for $p \leq q$, and by Holder's inequality:

$$
E_{p}\left(u_{q}\right) \leq\left(\frac{1}{c_{1}}\right)^{1 / p} c_{1}^{\frac{q-p}{q p}}\left(\int_{M}|R|^{q} \mathrm{~d} \mu_{q}\right)^{1 / q} \leq\left(\frac{1}{c_{1}}\right)^{1 / q}\left(\int_{M}|R|^{q} \mathrm{~d} \mu_{q}\right)^{1 / q}=E_{q}\left(u_{q}\right) .
$$


Hence, $\lim _{p \rightarrow \infty} E_{p}\left(u_{p}\right)=e_{\infty}$ exists, and from the lower semicontinuity of $E_{p}$ and the definition of lim inf, the relation

$$
E_{q}(u) \leq \liminf _{k \rightarrow \infty} E_{q}\left(u_{p_{k}}\right) \leq \liminf _{k \rightarrow \infty} E_{p_{k}}\left(u_{p_{k}}\right)=e_{\infty}
$$

follows. We also remark that $u$ belongs to $A^{\infty}\left(c_{1}, c_{2}, u_{0}\right)$ too. Indeed, the volume constraint (4) is preserved for $u_{p_{k}}$ as we pass to the limit, following the strong $C^{1}$ convergence that we have established in $\bar{M}$. The latter fact, along with our prescribed boundary values, guarantees that the average mean curvature constraint (6) is preserved too.

Letting $q \rightarrow \infty$ in (40), we conclude that $E_{\infty}(u) \leq e_{\infty}$. On the other hand, since $u_{p}$ minimizes $E_{p}$, we have:

$$
e_{\infty} \leq \lim _{p \rightarrow \infty} E_{p}\left(u_{p}\right) \leq \lim _{p \rightarrow \infty} E_{p}(\tilde{u})=E_{\infty}(\tilde{u}),
$$

for any other $\tilde{u} \in A^{\infty}\left(c_{1}, c_{2}, u_{0}\right)$. Now from (40) and (41), we conclude that $u$ is a minimizer for our problem in $A^{\infty}\left(c_{1}, c_{2}, u_{0}\right)$.

Further regularity results for $w$ may be established by working with equation (39) directly. In particular, we know that $u$ is uniformly bounded in $C^{1, \alpha}\left(M, g_{0}\right), \forall \alpha \in(0,1)$, via the Sobolev Embedding Theorem. Hence, the definition of the Laplace-Beltrami operator implies that its leading order coefficients belong to $C^{1, \alpha}(M), \forall \alpha \in(0,1)$. Also, $\frac{1}{n-1} R w$ belongs to $L^{\infty}$, by construction, so we may deduce that $w \in C^{1, \alpha}\left(M, g_{0}\right), \forall \alpha \in(0,1)$, using standard elliptic regularity theory. Iterating the latter result, and recalling that $R w=\gamma_{\infty}|w|$, we conclude, using Schauder Theory, that $w \in C^{2, \alpha}\left(M, g_{0}\right), \forall \alpha \in(0,1)$. But even more is true. Writing $w=w^{+}-w^{-}$, we may deduce that $w$ is locally smooth, on each one of the sets $M^{+}, M^{-}$, where $M^{+}=\{x \in M, w(x)>0\}$ and $M^{-}=\{x \in M, w(x)<0\}$. This follows easily after bootstrapping the existing Schauder estimates for $w$ on the sets $M^{+}$and $M^{-}$.

To conclude, it remains to prove that the set $\Gamma$ has the structure stated in Theorem 1 . For that we will use a result from [10], concerning the form and regularity of $\Gamma$. In particular, the following holds:

Proposition 3 The set $\Gamma=w^{-1}\{0\}$ is contained in the union of a countable union of embedded $C^{1, \rho}$ submanifolds and a countably $(n-2)$ rectifiable closed set.

Proof If $\alpha=0$, we have $w$ satisfying the equation

$$
-\Delta_{g} w-\frac{1}{n-1} R w=0,
$$

on $M$. We write $\Gamma=N(w) \cup S(w)$, where $N(w)=\{x \in \Gamma, D w \neq 0\}$ and $S(w)=\Gamma \backslash N(w)$. Then 0 is a regular value of $w$ for $x \in N(w)$, hence we can use the Implicit Function Theorem, to deduce that $N(w)$ is contained in the union of countably many $C^{2, \rho}$ manifolds of dimension $n-1$.

For $S(w)$, we use the fact that $w \in C^{2, \rho}(M)$, and then a result of Hardt and Simon [10] applies. Namely, we have $S(w)$ contained in a countable union of subsets of a pairwise disjoint collection of smooth $(n-2)$ dimensional submanifolds. Then, using a standard characterization for countably rectifiable sets, Lemma 11.1 from [18], we conclude that $S(w)$ is countably $(n-2)$ rectifiable.

Similarly, if $\alpha \neq 0$ the method used for $N(w)$ in the first part of the proof still applies, hence we only have to prove the corresponding result for $S(w)$. We have $w$ satisfying

$$
-\Delta_{g} w-\frac{1}{n-1} R w=\alpha,
$$


on $M$. Thus, $-\Delta_{g} w(x)=\alpha$ for $x \in S(w)$, and this implies that $\mathrm{d} \frac{\partial w}{\partial x_{i}}(x) \neq 0$, for some $i \leq n$, where $\left\{x_{i}\right\}$ are local coordinates centered around a point in $S(w)$. Consequently, $S(w)$ is contained in the union of countably many $(n-1)$ dimensional $C^{1, \rho}$ manifolds by the Implicit Function Theorem, since each $x \in S(w)$ is a regular value for $\frac{\partial w}{\partial x_{i}}$.

Hence, in any case we can conclude that $\mu_{0}(\Gamma)=0$, due to the structure of $\Gamma$, as presented in the last lemma. Then, it follows that $E(u)=\gamma_{\infty}$. Also, recall that $|R|=\gamma_{\infty}$ in $M \backslash \Gamma$. Thus, we have:

$$
|R|=E(u),
$$

in $M \backslash \Gamma$, finishing our proof.

Acknowledgements This work is based on my Ph.D. thesis at the University of Bath. I would like to thank my supervisor Prof. Roger Moser for his guidance, help and support.

Open Access This article is distributed under the terms of the Creative Commons Attribution 4.0 International License (http://creativecommons.org/licenses/by/4.0/), which permits unrestricted use, distribution, and reproduction in any medium, provided you give appropriate credit to the original author(s) and the source, provide a link to the Creative Commons license, and indicate if changes were made.

\section{References}

1. Aubin, T.: Equations differentielles non lineaires et probleme de Yamabe concernant la courbure scalaire. J. Math. Pures Appl. 55, 269-296 (1976)

2. Druet, O., Hebey, E., Robert, F.: Blow-up Theory for Elliptic PDEs in Riemannian Geometry. Princeton University Press, Princeton (2004)

3. Escobar, J.F., Schoen, R.M.: Conformal metrics with prescribed scalar curvature. Invent. Math. 86, 243254 (1986)

4. Escobar, J.F.: Conformal deformation of a Riemannian metric to a scalar flat metric with constant mean curvature on the boundary. Ann. Math. 2(136), 1-50 (1992)

5. Escobar, J.F.: The Yamabe problem on manifolds with boundary. J. Differ. Geom. 35(1), 21-84 (1992)

6. Evans, L.C.: Weak Convergence Methods for Nonlinear Partial Differential Equations, CBMS 74. American Mathematical Society, Providence (1974)

7. Gilbarg, D., Trudinger, N.S.: Elliptic Partial Differential Equations of Second Order. Springer, Heidelberg (1983)

8. Han, Z.C., Li, Y.Y.: The Yamabe problem on manifolds with boundary: existence and compactness results. Duke Math. J. 99(3), 489-542 (1999)

9. Han, Q., Lin, F.: Elliptic partial differential equations, CIMS Lecture Notes, Courant Institute of Mathematical Sciences, vol. 1, 2nd edn. American Mathematical Society, Providence (2000)

10. Hardt, R., Simon, L.: Nodal sets for solutions of elliptic equations. J. Differ. Geom. 30, 505-522 (1989)

11. Kazdan, J.L., Warner, F.W.: Curvature functions for compact 2-manifolds. Ann. Math. 99(2), 14-47 (1974)

12. Lee, J.M., Parker, T.H.: The Yamabe problem. Bull. Am. Math. Soc. (N.S.) 17(1), 37-91 (1987)

13. Marques, F.C.: Existence results for the Yamabe problem on manifolds with boundary. Indiana Univ. Math. J. 54(6), 1599-1620 (2005)

14. Marques, F.C.: Conformal deformations to scalar-flat metrics with constant mean curvature on the boundary. Commun. Anal. Geom. 15(2), 381-405 (2007)

15. Moser, R., Schwetlick, H.: Minimizers of a weighted maximum of the Gauss curvature Ann. Global Anal. Geom. 41, 199-207 (2012)

16. Schoen, R.: Conformal deformation of a Riemannian metric to constant scalar curvature. J. Differ. Geom. 20, 479-495 (1984)

17. Schwetlick, H., Struwe, M.: Convergence of the Yamabe flow for "large" energies. J. Reine Angew. Math. 562, 59-100 (2003)

18. Simon, L.: Lectures on geometric measure theory. In: Proceedings of the Centre for Mathematical Analysis. Australian National University, Australia (1983) 
19. Trudinger, N.: Remarks concerning the conformal deformation of Riemannian structures on compact manifolds. Ann. Scuola Norm. Sup. Pisa 22, 265-274 (1968)

20. Yamabe, H.: On a deformation of Riemannian structures on compact manifolds. Osaka Math. J. 12, 21-37 (1960) 